Article

\title{
Comparative Analysis of the Transcriptomes of Persisting and Abscised Fruitlets: Insights into Plant Hormone and Carbohydrate Metabolism Regulated Self-Thinning of Pecan Fruitlets during the Early Stage
}

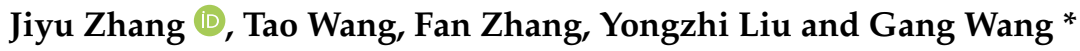

check for

updates

Citation: Zhang, J.; Wang, T.; Zhang,

F.; Liu, Y.; Wang, G. Comparative

Analysis of the Transcriptomes of

Persisting and Abscised Fruitlets:

Insights into Plant Hormone and

Carbohydrate Metabolism Regulated

Self-Thinning of Pecan Fruitlets

during the Early Stage. Curr. Issues

Mol. Biol. 2022, 44, 176-193. https://

doi.org/10.3390/cimb44010013

Academic Editor: Vijai Bhadauria

Received: 8 November 2021

Accepted: 27 December 2021

Published: 30 December 2021

Publisher's Note: MDPI stays neutral with regard to jurisdictional claims in published maps and institutional affiliations.

Copyright: (C) 2021 by the authors. Licensee MDPI, Basel, Switzerland. This article is an open access article distributed under the terms and conditions of the Creative Commons Attribution (CC BY) license (https:// creativecommons.org/licenses/by/ $4.0 /)$.
Institute of Botany, Jiangsu Province and Chinese Academy of Sciences, Nanjing 210014, China; maxzhangjy@163.com (J.Z.); immmorer@163.com (T.W.); mumizhongfeng@126.com (F.Z.); liuyz1965@163.com (Y.L.)

* Correspondence: wg20092011@163.com; Tel.: +86-025-8434-7033
Abstract: Pecan is one of the most popular nut species in the world. The fruit drop rate of the pecan 'Pawnee' is more than $57 \%$, with four fruit drop stages, which is very serious. In this study, we conducted transcriptomic profiling of persisting and abscised fruitlets in early fruit development by RNA-seq. A total of 11,976 differentially expressed genes (DEGs) were identified, 3012 upregulated and 8964 downregulated, in a comparison of abscised vs. persisting fruitlets at 35 days after anthesis (DAA). Our transcriptomic data suggest that gene subsets encoding elements involving the biosynthesis, metabolism, perception, signal transduction, and crosstalk of the plant hormones abscisic acid (ABA), auxin, cytokinin, ethylene, and gibberellin (GA) and plant growth regulators jasmonates, salicylic acid, and brassinosteroids were differentially expressed. In addition, the majority of transcriptionally activated genes involved in hormone signaling (except for ethylene and salicylic acid signaling) were downregulated in abscised fruitlets. The differential expression of transcripts coding for enzymes involved in sucrose, glucose, trehalose, starch, galactose, and galactinol metabolism shows that sucrose, galactinol, and glucose synthesis and starch content were reduced as starch biosynthesis was blocked, and retrogradation and degradation intensified. These results suggest that the abscised pecan fruitlets stopped growing and developing for some time before dropping, further indicating that their sugar supply was reduced or stopped. The transcriptome characterization described in this paper contributes to unravelling the molecular mechanisms and pathways involved in the physiological abscission of pecan fruits.

Keywords: pecan; abscised fruitlet; plant hormone; carbohydrate metabolism

\section{Introduction}

Vegetative and reproductive organs that are senescent, infected, or damaged are shed from the main plant body, which is called abscission. Abscission is a specific sequence of highly complex regulated events [1]. The regulatory network activated by the abscising organ leads to the activation of abscission zones. To simplify the explanation, the abscission process includes four phases. The first step is the development of an abscission zone. The next step is the activation of abscission signaling. Then, enzymatic hydrolysis takes place in the middle lamella of the abscission zone (AZ), and AZ cells begin to enlarge. The last step is the further differentiation 1and sealing of the abscission scar [2].

Fruitlet abscission, especially for the so-called physiological drop or June drop, is very common in fruit tree development in order to control fruit load according to the nutritional status, allowing the plant to make efficient use of resources [3]. The molecular mechanisms that control and lead to early physiological fruitlet abscission are studied in model apple [3-6] and tomato [7,8] plants. The progress of physiological drop differs from 
senescence-driven abscission at or after ripening. Dropping of young fruits during early phases of development can be understood as a developmental response by which the plant selectively abscises fruitlets that represent weaker sinks to adapt to nutritional shortages [5]. The molecular mechanisms regulating early fruit development and the plasticity of fruit development in response to endogenous and environmental changes can be understood by studying fruit physiological drop in this scenario.

Fruit set is the process of ovary tissues undergoing transformation into fruit. It is well known that auxins and gibberellins (GAs) play a critical role in the inductive phase of fruit set. A few studies have shown that auxins trigger cell division, and their interplay with GAs sustain cell expansion [9]. However, the transformation of the ovary into fruit of tomato and Arabidopsis is prevented by a negative control exerted by auxin/indole acetic acid (AUX/IAA) and auxin response factor (ARF) proteins. This negative regulation can be removed through pollination/fertilization or treatment with auxins, leading to cell proliferation and fruit set. Ethylene and abscisic acid (ABA) biosynthesis and action appear to be significantly downregulated, and concomitantly those of auxin and GA biosynthesis and action are activated as soon as fruit set is triggered $[10,11]$. This suggests that plant hormones play an important role in fruit development. A few reports have shown that plant hormones auxin, ethylene, and ABA seem to play major roles, and GAs, cytokinins, and jasmonates have also been reported to be involved $[3,12,13]$.

Sugar metabolism also plays a major role in the response to the progress of fruitlet abscission. The contents of sorbitol, glucose, fructose, and sucrose were shown to be lower in abscising fruitlets than in persisting fruitlets [6]. Transcriptomic analysis showed that genes coding trehalose-6-phosphate synthase, sorbitol transporter, UDP-glucosyltransferases, and UDP-Glc-4-epimerase were upregulated in abscising apple fruitlet [5]. The class of enzyme genes is also controlled by sugar starvation and involved in resource mobilization in other species [14-16].

Pecans (Carya illinoinensis (Wangenh.) K. Koch), which are native to North America and belong to the Juglandaceae family, are the most economically valuable nut trees in the world $[17,18]$. Pecan trees exhibit a strong tendency to produce a heavy crop one year and a light crop the following year. It is sometimes difficult to compile cultivation recommendations to minimize alternate bearing. Despite years of research, the physiology behind alternate bearing is only partially understood, and this phenomenon remains the principal challenge faced by the pecan industry year after year. It is very important to make clear the process of fruit development and the mechanism of natural fruit abscission in pecan in order to control a reasonable load. Thus, transcriptomes from persisting and abscised fruitlets were sequenced and differentially expressed genes between them were identified in order to fully understand the differences in gene expression and explore the cause of fruitlet abscission in pecan. The contents of plant hormones of persisting and abscised fruitlets were measured in this study. This study can serve to broaden our understanding of the mechanism of fruit development and natural fruit abscission.

\section{Experimental Section}

\subsection{Plant Materials, Fruit Development, and Drop Dynamics Analysis}

Experiments were carried out over 2 years on 10-year-old pecan trees (Pawnee) with spacing of $6 \mathrm{~m} \times 8 \mathrm{~m}$, grown with standard horticultural practices at the experimental farm of the Institute of Botany, Jiangsu Province, and Chinese Academy of Sciences $\left(32^{\circ} 18^{\prime} \mathrm{N}\right.$; $118^{\circ} 52^{\prime}$ E). Fruit drop was monitored from 7 DAA until fruit maturity at weekly intervals using 50 pre-marked fruit clusters from each of 5 homogeneous trees. Further, 10 fruits were sampled from each of 5 additional homogeneous trees from 7 DAA until fruit maturity at weekly intervals to study fruit development. Finally, 10 naturally abscised and persisting fruitlets were harvested at 36 and 48 DAA from each of 3 homogeneous trees for the study reported in this paper. 


\subsection{RNA Isolation and cDNA Library Preparation and Sequencing}

Naturally abscised and persisting fruitlets harvested at 36 DAA were used for transcriptome analysis. Total RNA was extracted from the fruitlets using a Trizol reagent kit (Invitrogen, Carlsbad, CA, USA) according to the manufacturer's protocol. The individual RNA samples were analyzed spectrophotometrically for protein contamination, and the samples with A260/ A280 ratio values ranging from 1.9 to 2.0 were chosen. After total RNA was extracted, mRNA was enriched by oligo (dT) beads. The enriched mRNA was then fragmented into short fragments and reverse transcribed into cDNA by using the NEBNext Ultra RNA Library Prep Kit for Illumina (NEB\#E7530, New England Biolabs, Ipswich, MA, USA). The purified double-stranded cDNA fragments were end repaired, poly(A) was added, and they were ligated to Illumina sequencing adapters. The ligation reaction was purified with AMPure XP Bead $(1.0 \times)$. Ligated fragments were subjected to size selection by agarose gel electrophoresis and amplified PCR. The resulting cDNA library was sequenced using an Illumina HiSeq 2500 by Genedenovo Biotechnology Co., Ltd. (Guangzhou, China). The raw sequencing data generated from this study have been deposited in NCBI SRA (http:/ / www.ncbi.nlm.nih.gov/sra, last accessed on 1 December 2021) under accession number PRJNA784729.

\subsection{Analysis of Differential Gene Expression}

Clean reads were obtained by filtering the raw reads, including adapters or lowquality bases, by fastp (v.18) [19]. The clean reads were mapped to a ribosome RNA database using the Bowtie2 (v.2.28) short read alignment tool [20] to identify and remove residual rRNA reads. The remaining clean reads were mapped to the pecan genome (Cil.genome.fa, ftp:/ / parrot.genomics.cn/gigadb/pub/10.5524/100001_101000/100571/) using HISAT2.2.4 [21] with the parameter "-rna-strandness RF" and default settings for the other parameters. The unmapped reads were assembled by using StringTie v.1.3.1 [22], and the assembled sequences were regarded as novel genes. HTSeq (v.0.6.0) [23] was used to calculate the raw read counts for each gene. The gene expression was calculated and normalized to reads per kilobase per million mapped reads (RPKM) [24]. Analysis of gene expression differences between two groups was performed by DESeq2 software (European Molecular Biology Laboratory, Heidelberg, Germany) [25]. Transcripts with a false discovery rate (FDR) $<0.05$ and absolute fold change $>2$ were defined as differentially expressed genes (DEGs). Gene Ontology (GO) enrichment ( $p$-value $<0.05$ ) was studied by running all DEGs through the GO database (http: / / www.geneontology.org/) to further classify the genes or their products into terms (molecular function, biological process, and cellular component) to understand their biological functions. Pathway projects were performed according to the KEGG pathway database for pathway enrichment analysis of DEGs.

\subsection{Plant Hormone Content Analysis}

Approximately $200 \mathrm{mg}$ of normal persisting fruitlets and abscised fruitlets (just shed from the main plant body) were used to measure the levels of plant hormones (ABA, IBA, GA, and TZR). Plant hormones were detected by high-performance liquid chromatography coupled with electrospray ionization tandem mass spectrometry (HPLC-ESI-MS/MS) on an Agilent 1290 HPLC (Agilent Technologies Inc., Santa Clara, CA, USA) and a Sciex QTRAP 6500 mass spectrometer (AB Sciex LLC, Framingham, MA, USA). The experiment was performed by Nanjing Innovation Biotechnology Co., Ltd. (Nanjing, China). Three biological replicates were analyzed for this experiment, each one containing 30 fruitlets. Statistically significant differences of plant hormones were calculated with GraphPad Prism 7.0 software (San Diego, CA, USA). 


\section{Results}

\subsection{Analysis of Pecan Fruit Drop Dynamics}

Pecan 'Pawnee' fruit drop and fruit development dynamics were investigated in this study (Figure 1). The results show that fruit growth exhibited the characteristic of a single sigmoid growth curve, presenting only one rapid growth period. The fruit drop rate was more than 57\%, with four fruit drop stages, occurring at 0-28 days after anthesis (DAA), 28-49 DAA, 49-77 DAA, and 77-104 DAA. The fruit drop ratio was the largest at the third fruiting stage, accompanied by a rapid fruit growth period. The single fruit mass of persisting fruitlets was about twice that of abscised fruitlets (just shed from the main plant body) (Figure 1 and S1), indicating that the abscised fruitlets had stopped growing and developing for some time before dropping.

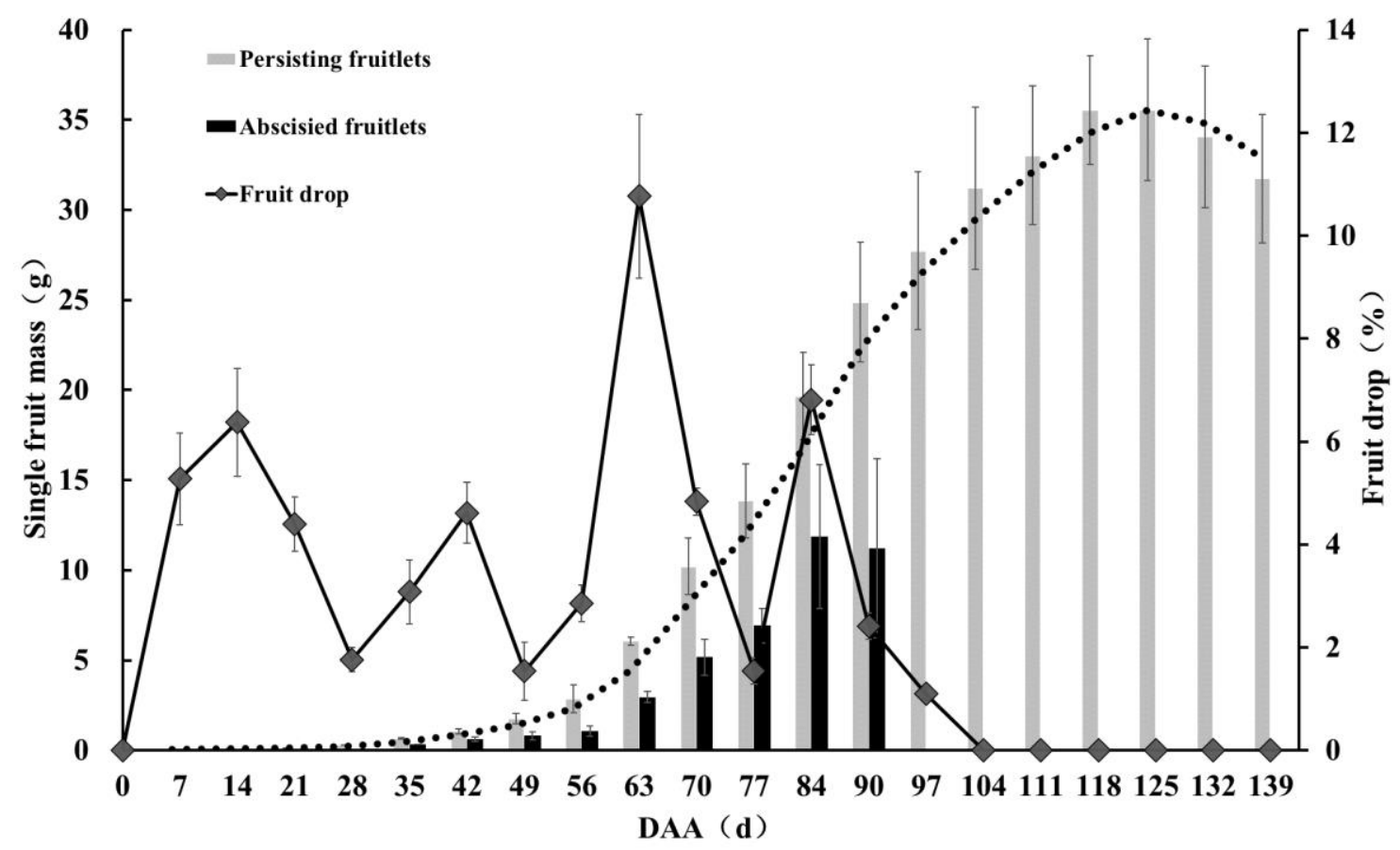

Figure 1. Analysis of pecan 'Pawnee' fruit development and drop dynamics. Black dot line indicates the fruit development dynamic trend. Mean values $( \pm S D)$ of three biological replicates are shown. DAA, days after anthesis.

3.2. RNA Sequencing of Transcriptomes of Persisting and Abscised Fruitlets and Mapping of RNA Sequences to Reference Genome

To test the differentially expressed genes of persisting fruitlets (P1) and abscised fruitlets (A1) in the early stage of fruit development (35 DAA), two groups with six samples were analyzed by RNA-seq, and a total of 35.66 GB of clean data was generated by strict quality control and processing (Supplementary Table S1). Details on data and data quality before and after filtering are shown in Table 1 and Supplementary Table S1. In all, 112, 434, and 202 reads and 135, 904, and 652 reads were generated for abscised and persisting fruitlets, respectively, after filtering out duplicate sequences and ambiguous and low-quality reads (Supplementary Table S1). The numbers of clean bases and reads of persisting fruitlets were more than those of abscised fruitlets (Table 1 and Supplementary Table S1). The average GC percentage was $45.41 \%$, with a QC30 base percentage above $93.05 \%$. HQ clean reads were mapped to the pecan reference genome (Cil.genome.fa). Approximately 38.94 million clean reads $\left(94.11 \%\right.$ of the total) were mapped, and the number of mapped reads $\left(42.57 \times 10^{6}\right)$ of persisting fruitlets was more than that of abscised fruitlets $\left(35.32 \times 10^{6}\right)$. This indicates that the number of transcripts of persisting fruitlets was more than that of abscised fruitlets. A total of 28,624 genes were obtained from 6 transcriptome libraries, including 26,537 reference 
genes and 2087 novel genes (Supplementary Table S2). The numbers of reference genes, novel genes, and total genes of persisting fruitlet transcripts were more than those of abscised fruitlet transcripts (Table 1 and Supplementary Table S1).

Table 1. Sequencing and statistics of transcriptome data of two groups with reference genome (Cil.genome.fa).

\begin{tabular}{ccc}
\hline Group Name & Persisting Fruitlets (P1) & Abscised Fruitlets (A1) \\
\hline No. of clean bases $\left(\times 10^{8}\right)$ & $68.24 \pm 1.00$ & $56.40 \pm 0.69$ \\
No. of total reads $\left(\times 10^{6}\right)$ & $45.30 \pm 0.68$ & $37.48 \pm 0.47$ \\
No. of mapped reads $\left(\times 10^{6}\right)$ & $42.57 \pm 0.65$ & $35.32 \pm 0.40$ \\
Mapped percentage $(\%)$ & $93.98 \pm 0.0 .3$ & $94.25 \pm 0.16$ \\
No. of unique mapped reads $\left(\times 10^{6}\right)$ & $41.45 \pm 0.62$ & $34.39 \pm 0.47$ \\
No. of sequenced reference genes & $24,307 \pm 73$ & $21,723 \pm 360$ \\
Percentage of sequenced reference & $78.23 \pm 0.24$ & $69.91 \pm 1.16$ \\
genes $(\%)$ & $1874.00 \pm 14.73$ & $1701.33 \pm 22.68$ \\
No. of sequenced novel genes & $89.80 \pm 0.71$ & $81.52 \pm 1.09$ \\
Percentage of sequenced novel genes $(\%)$ & $26,181.00 \pm 88.10$ & $23,424.33 \pm 382.98$ \\
Sequenced total genes & $78.96 \pm 0.27$ & $70.64 \pm 1.15$ \\
Percentage of sequenced total genes $(\%)$ & \\
\hline
\end{tabular}

\subsection{Differentially Expressed Gene Analysis}

In our study, we calculated the correlation coefficient between the samples to test sample repeatability. The correlation coefficient in the repeat group was greater than 0.808 (Supplementary Figure S2), indicating consistency among the three biological replicates. Thus, the RNA-seq results were confirmed to be highly reliable for further analysis. A total of 11,976 DEGs (about $41.84 \%$ of total sequenced genes) were identified in the comparison of abscised fruitlets vs. persisting fruitlets at 35 DAA; among them, 3012 DEGs $(25.15 \%)$ were upregulated and 8964 DEGs $(74.85 \%)$ were downregulated (Supplementary Tables S3 and S4). There were about three times as many downregulated genes as upregulated genes, indicating that the vital signs of abscised fruitlets had diminished.

Gene Ontology (GO) functional classification included three GO trees (cellular components, molecular functions, and biological processes) and 48 functional groups (Figure 2 and Supplementary Table S5). In the category of biological processes, the largest groups were metabolic, cellular, and single-organism processes. For the cellular components, DEGs with cell, cell part, membrane, and organelle formed the major groups. Catalytic activity and binding were the dominant groups in the molecular function category. The top five enrichment categories in GO were catalytic activity, cellular process, single-organism process, primary metabolic process, and single-organism cellular process (Supplementary Table S6). In order to understand their biological function, all of the DEGs were also mapped to terms in the Kyoto Encyclopedia of Genes and Genomes (KEGG) database. Finally, 1050 DEGs were matched and assigned to 129 KEGG pathways (Supplementary Table S7). The first five biological pathways, involving biosynthesis of secondary metabolites, metabolic pathways, plant-pathogen interaction, monoterpenoid biosynthesis, and starch and sucrose metabolism, were significantly enriched in persisting fruitlets (P1) and abscised fruitlets (A1) (Figure 3 and Table 2). 


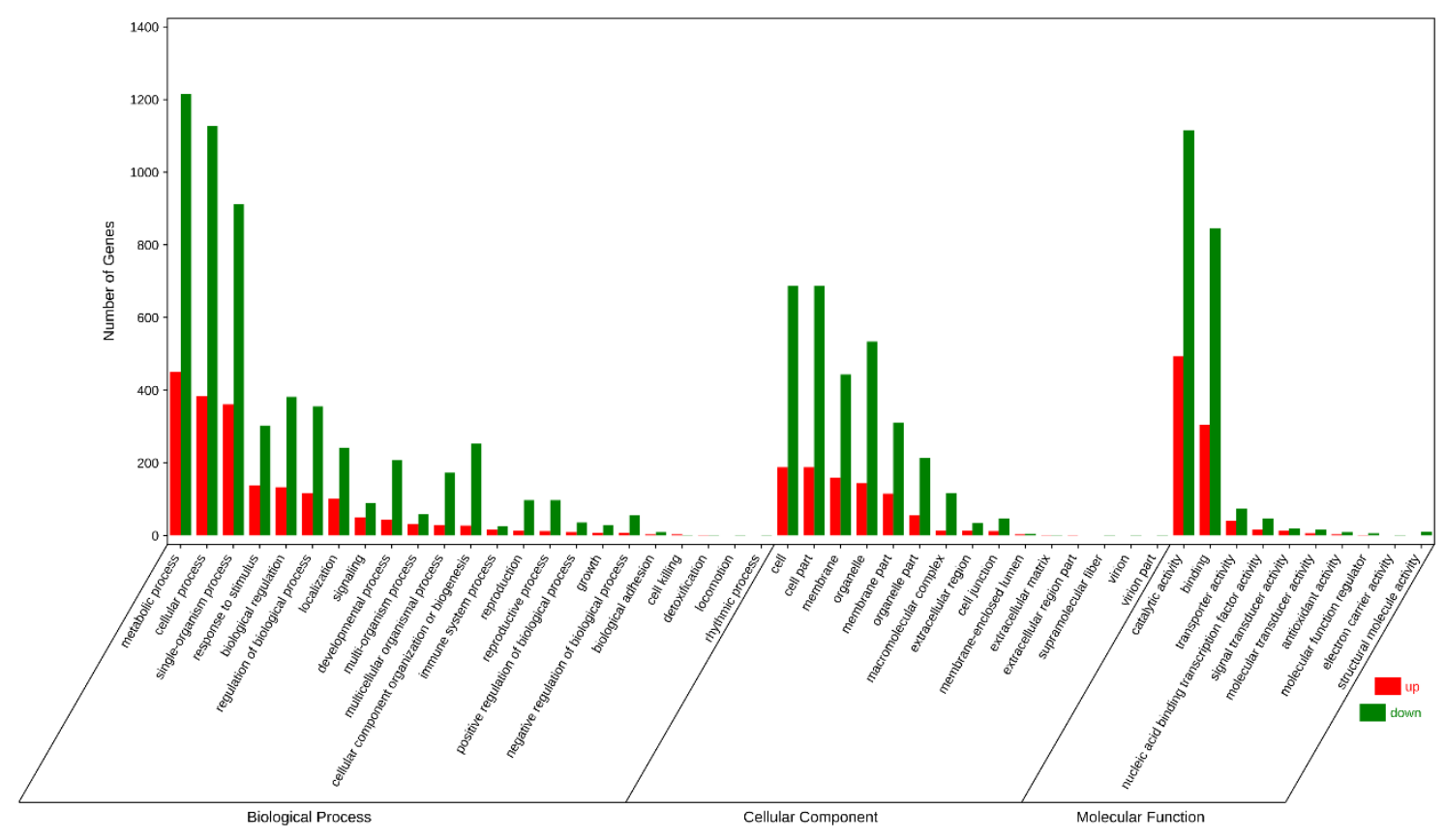

Figure 2. GO functional classification annotation of differentially expressed genes of pecan in comparison of abscised fruitlets vs. persisting fruitlets at 35 DAA.

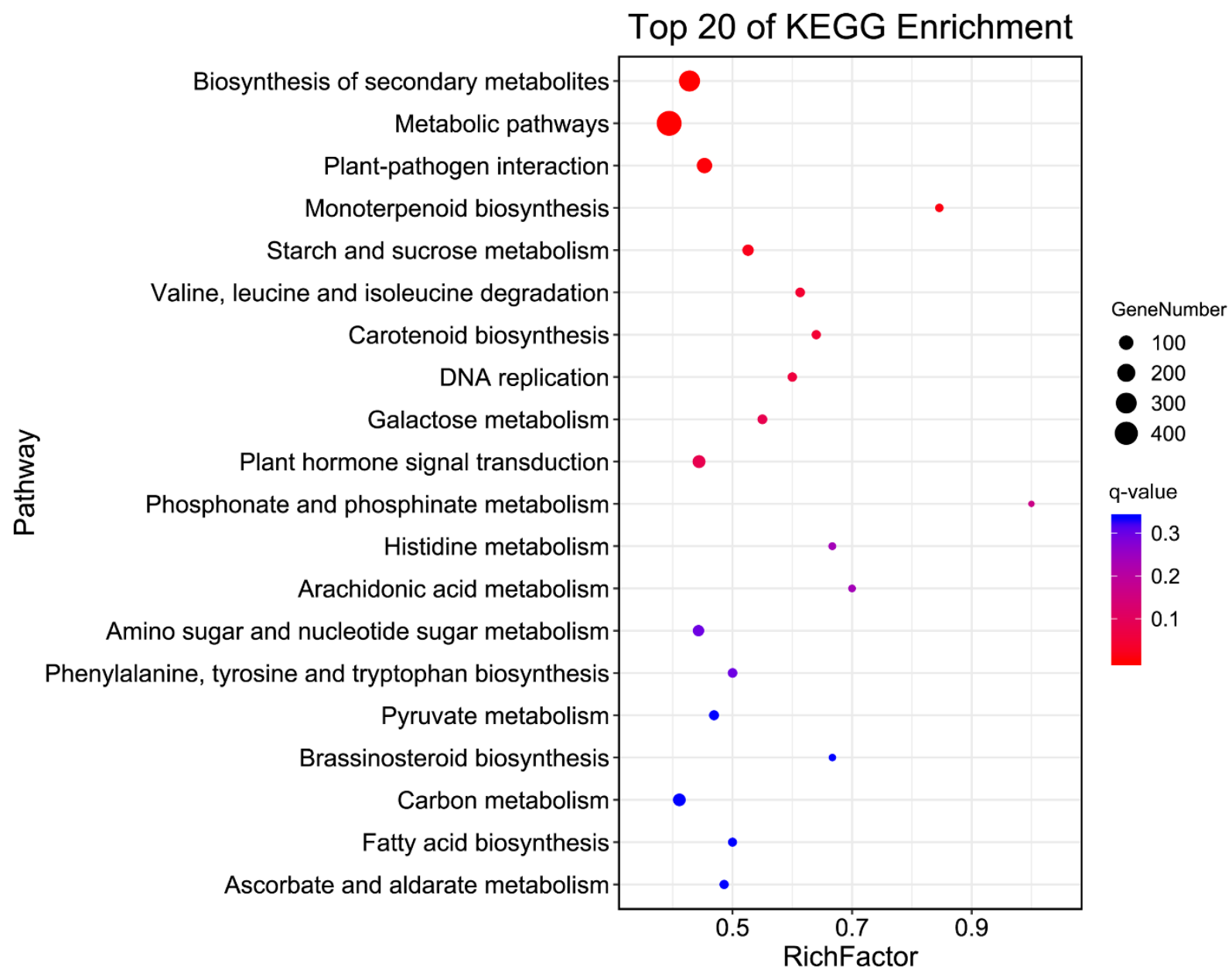

Figure 3. Top KEGG pathways mapping enriched differential progress. 
Table 2. KEGG pathways mapping enriched differential progress $(p<0.01)$.

\begin{tabular}{ccccc}
\hline Pathway & DEGs & $p$-Value & q-Value & Pathway ID \\
\hline Biosynthesis of secondary metabolites & 306 & $8.64 \times 10^{-8}$ & $1.11 \times 10^{-5}$ & ko01110 \\
Metabolic pathways & 492 & $1.50 \times 10^{-6}$ & $9.69 \times 10^{-5}$ & ko01100 \\
Plant-pathogen interaction & 120 & $9.58 \times 10^{-5}$ & 0.004 & ko04626 \\
Monoterpenoid biosynthesis & 11 & $2.94 \times 10^{-4}$ & 0.009 & ko00902 \\
Starch and sucrose metabolism & 41 & $6.91 \times 10^{-4}$ & 0.018 & ko00500 \\
Valine, leucine, and isoleucine & 19 & $1.99 \times 10^{-3}$ & 0.043 & ko00280 \\
degradation & 16 & $2.40 \times 10^{-3}$ & 0.044 & ko00906 \\
Carotenoid biosynthesis & 18 & $3.64 \times 10^{-3}$ & 0.059 & ko03030 \\
DNA replication & 22 & $5.96 \times 10^{-3}$ & 0.083 & ko00052 \\
Galactose metabolism & 67 & $6.40 \times 10^{-3}$ & 0.083 & ko04075 \\
Plant hormone signal transduction & & & &
\end{tabular}

\subsection{Identification of Differentially Expressed Proteins Involved in Plant Hormone Signal Transduction}

Gene subsets encoding elements involved in hormone biosynthesis, metabolism, perception, signal transduction, and crosstalk were found to be differentially expressed in the comparison of abscised fruitlets vs. persisting fruitlets at 35 DAA using de novo transcriptome sequencing (Table 3 and Supplementary Table S8). The statistical analyses were performed for plant hormones ABA, auxin, cytokinin, ethylene, and GA and plant growth regulators jasmonates, salicylic acid, and brassinosteroids. All 3 identified DEGs of the ABA metabolic process, 7 DEGs in response to ABA, and 8 out of 9 DEGs involved in ABA signal transduction were downregulated in abscised fruitlets vs. persisting fruitlets. All 6 identified DEGs involved in the auxin metabolic process, 7 out of 10 DEGs involved in auxin transport, and 15 out of 20 DEGs involved in auxin signal transduction were downregulated. Half of the identified DEGs involved in the gibberellin metabolic process and 10 out of 12 DEGs involved in cytokinin signal transduction were downregulated. Two out of 3 identified DEGs involved in the gibberellin metabolic process, all 4 DEGs involved in the gibberellin-mediated signaling pathway, and 2 out of 3 DEGs involved in gibberellin signal transduction were downregulated. All 3 identified DEGs involved in ethylene signal transduction were upregulated. These results show that almost all identified DEGs involved in ABA, auxin, cytokinin, and gibberellin were downregulated, and all identified DEGs involved in ethylene were upregulated. Eleven out of 20 identified DEGs involved in the SA metabolic process and 5 out of 6 DEGs involved in SA signal transduction were upregulated, but, all 9 identified DEGs involved in the JA metabolic process and 5 out of 7 DEGs involved in JA signal transduction were downregulated, indicating that SA biosynthesis and the signal transduction pathway were promoted, but were suppressed in abscised fruitlets of pecan.

Table 3. Identified differentially expressed proteins involved in plant hormone signal transduction (ko04075).

\begin{tabular}{cccccc}
\hline Gene ID & Description & Symbol & Name & $\log 2(A / P)$ & $p$-Value \\
\hline CIL1308S0009 & Abscisic acid & & & & \\
CIL1506S0033 & Abscisic acid receptor PYL9 & PYR/PYL & PYL9 & -1.938 & $4.18 \times 10^{-15}$ \\
CIL1317S0086 & Protein phosphatase 2C 53 & PP2C & HAB1 & -2.868 & $6.3 \times 10^{-9}$ \\
CIL1562S0028 & Protein phosphatase 2C 16 & PP2C & HAB1 & -1.024 & 0.029075 \\
CIL0302S0016 & Protein phosphatase 2C 75 & PP2C & AHG1 & -2.910 & $2.89 \times 10^{-8}$ \\
CIL0409S0006 & Serine/threonine-protein kinase SAPK7 & SnRK2 & SRK2H & -2.478 & $6.01 \times 10^{-8}$ \\
CIL0045S0005 & Serine / threonine-protein kinase SAPK2 & SnRK2 & SAPK2 & -7.128 & $2.1 \times 10^{-9}$ \\
CIL1371S0045 & ABSCISIC ACID-INSENSITIVE 5-like protein 5 & ABF & DPBF3 & 2.287 & $1.66 \times 10^{-59}$ \\
CIL1387S0048 & ABSCISIC ACID-INSENSITIVE 5-like protein 6 & ABF & ABF2 & -2.671 & $1.13 \times 10^{-6}$ \\
\hline & ABSCISIC ACID-INSENSITIVE 5-like protein 2 & ABF & DPBF3 & -1.484 & 0.000172 \\
\hline
\end{tabular}


Table 3. Cont.

\begin{tabular}{|c|c|c|c|c|c|}
\hline Gene ID & Description & Symbol & Name & $\log 2(\mathrm{~A} / \mathrm{P})$ & $p$-Value \\
\hline \multicolumn{6}{|c|}{ Auxin } \\
\hline CIL1565S0004 & Auxin transporter-like protein 3 & AUX1 & LAX3 & 1.772 & $1.09 \times 10^{-7}$ \\
\hline CIL1464S0004 & TRANSPORT INHIBITOR RESPONSE protein & TIR & TIR1 & -2.584 & $3.62 \times 10^{-6}$ \\
\hline CIL0202S0015 & Auxin-responsive protein IAA16-like & AUX/IAA & IAA16 & -16.109 & $3.02 \times 10^{-23}$ \\
\hline CIL0203S0027 & Auxin-responsive protein IAA9-like & AUX/IAA & IAA9 & -3.587 & $1.04 \times 10^{-39}$ \\
\hline CIL0344S0014 & Auxin-responsive protein IAA18-like & AUX/IAA & IAA26 & -2.671 & $2.16 \times 10^{-11}$ \\
\hline CIL0732S0001 & Auxin-responsive protein IAA9-like & AUX/IAA & IAA9 & -10.617 & 0.000112 \\
\hline CIL1268S0077 & Auxin-responsive protein IAA27-like & AUX/IAA & IAA8 & -3.986 & $6.35 \times 10^{-5}$ \\
\hline CIL1294S0084 & Auxin-responsive protein IAA20-like & AUX/IAA & IAA20 & -9.765 & 0.001964 \\
\hline CIL1320S0049 & Auxin-responsive protein IAA27-like & AUX/IAA & IAA27 & -4.217 & $2.06 \times 10^{-11}$ \\
\hline CIL1358S0014 & Auxin-responsive protein IAA29-like & AUX/IAA & IAA11 & -10.951 & $8.53 \times 10^{-7}$ \\
\hline CIL1490S0013 & Auxin response factor 18 & ARF & ARF9 & -8.323 & $1.83 \times 10^{-54}$ \\
\hline CIL1564S0002 & Auxin response factor 9 & $\mathrm{ARF}$ & ARF9 & -3.530 & $1.08 \times 10^{-38}$ \\
\hline CIL1354S0026 & Auxin response factor 19 & $\mathrm{ARF}$ & ARF7 & -2.515 & $3.06 \times 10^{-13}$ \\
\hline CIL1313S0055 & Probable indole-3-acetic acid-amido synthetase GH3.6 & GH3 & GH3.6 & 2.440 & $5.86 \times 10^{-18}$ \\
\hline CIL1405S0075 & Indole-3-acetic acid-amido synthetase GH3.6 & $\mathrm{GH} 3$ & GH3.6 & -4.055 & $6.98 \times 10^{-7}$ \\
\hline CIL1456S0019 & Probable indole-3-acetic acid-amido synthetase GH3.1 & GH3 & GH3.1 & -2.721 & $1.89 \times 10^{-6}$ \\
\hline CIL1295S0016 & Auxin-responsive protein SAUR36 & SAUR & SAUR36 & 3.508 & $3.6 \times 10^{-26}$ \\
\hline CIL0367S0005 & Auxin-induced protein $\mathrm{X} 15$ & SAUR & SAUR50 & -3.078 & 0.003923 \\
\hline MSTRG.7503 & Auxin-responsive protein SAUR32 & SAUR & SAUR32 & 6.343 & $4.96 \times 10^{-90}$ \\
\hline CIL1294S0056 & Indole-3-acetic acid-induced protein ARG7 & SAUR & SAUR36 & 10.129 & 0.000393 \\
\hline \multicolumn{6}{|c|}{ Cytokinin } \\
\hline CIL1595S0016 & Histidine kinase 2 & CRE1 & AHK2 & -4.205 & $3.31 \times 10^{-19}$ \\
\hline CIL1384S0015 & Histidine kinase 2 & CRE1 & AHK2 & -1.934 & 0.004026 \\
\hline CIL0037S0021 & Histidine-containing phosphotransfer protein 1 & AHP & AHP1 & -12.078 & $3.11 \times 10^{-9}$ \\
\hline CIL1268S0039 & Histidine-containing phosphotransfer protein 4 & AHP & PHP5 & -6.299 & $2.73 \times 10^{-5}$ \\
\hline CIL1268S0040 & Histidine-containing phosphotransfer protein 4 & AHP & AHP4 & 10.326 & $1.02 \times 10^{-6}$ \\
\hline CIL1369S0025 & Histidine-containing phosphotransfer protein 1 & AHP & AHP1 & -1.748 & $3.06 \times 10^{-9}$ \\
\hline MSTRG.14062 & Histidine-containing phosphotransfer protein 1 & AHP & AHP1 & 1.101 & 0.001041 \\
\hline CIL1575S0022 & Two-component response regulator ARR12 & B-ARR & RR23 & -2.035 & $5.52 \times 10^{-13}$ \\
\hline CIL0004S0012 & Two-component response regulator ARR8 & A-ARR & ARR8 & -11.423 & 0.000187 \\
\hline CIL0338S0017 & Two-component response regulator ARR9 & A-ARR & ARR9 & -1.943 & 0.030964 \\
\hline CIL1308S0026 & Two-component response regulator ARR5 & A-ARR & ARR4 & -4.320 & $2.19 \times 10^{-12}$ \\
\hline CIL1596S0045 & Two-component response regulator ARR5 & A-ARR & ARR15 & -5.520 & $3.19 \times 10^{-12}$ \\
\hline \multicolumn{6}{|c|}{ Ethylene } \\
\hline CIL1354S0046 & Ethylene-responsive transcription factor $1 \mathrm{~B}$ & $\mathrm{EBF} 1 / 2$ & ERF1B & 2.436 & 0.000283 \\
\hline CIL1358S0043 & EIN3-binding F-box protein 1 & $\mathrm{EBF} 1 / 2$ & EBF2 & 2.280 & $7.01 \times 10^{-36}$ \\
\hline CIL1493S0026 & Ethylene receptor 2 & ETR & ETR2 & 4.072 & $1.15 \times 10^{-73}$ \\
\hline \multicolumn{6}{|c|}{ Gibberellin } \\
\hline CIL1324S0067 & DELLA protein SLN1 & DELLA & GAI1 & -3.061 & $1.6 \times 10^{-16}$ \\
\hline CIL1294S0080 & Transcription factor PIF3 & $\mathrm{TF}$ & PIL15 & -5.458 & $8.24 \times 10^{-14}$ \\
\hline CIL1495S0010 & Transcription factor PIF1 & $\mathrm{TF}$ & PIF1 & 2.298 & $5.46 \times 10^{-8}$ \\
\hline \multicolumn{6}{|c|}{ Brassinosteroid } \\
\hline CIL1492S0038 & $\begin{array}{l}\text { BRASSINOSTEROID INSENSITIVE 1-associated } \\
\text { receptor kinase } 1\end{array}$ & BAK1 & BAK1 & 1.609 & $1.12 \times 10^{-22}$ \\
\hline CIL1312S0044 & Brassinosteroid LRR receptor kinase & BRI1 & CURL3 & -8.773 & $1.46 \times 10^{-58}$ \\
\hline MSTRG.23225 & Serine/threonine-protein kinase & BSK & BSK1 & -1.699 & 0.003421 \\
\hline CIL1383S0002 & BES1/BZR1 homolog protein 2 & $\mathrm{BZR} 1 / 2$ & $\mathrm{BEH} 2$ & -1.441 & 0.013346 \\
\hline CIL0182S0015 & HMA domain-containing protein & $\mathrm{BZR} 1 / 2$ & - & 5.157 & 0.000157 \\
\hline CIL1577S0019 & Xyloglucan endotransglucosylase/hydrolase protein 22 & $\mathrm{TCH} 4$ & XTH22 & -3.772 & $2.16 \times 10^{-5}$ \\
\hline CIL1371S0059 & Cyclin-D3-1 & CYCD3 & CYCD3-3 & -11.257 & $4 \times 10^{-9}$ \\
\hline
\end{tabular}


Table 3. Cont.

\begin{tabular}{|c|c|c|c|c|c|}
\hline Gene ID & Description & Symbol & Name & $\log 2(\mathrm{~A} / \mathrm{P})$ & $p$-Value \\
\hline \multicolumn{6}{|c|}{ JA } \\
\hline CIL1295S0055 & Protein TIFY 10A & JAZ & TIFY10B & 2.236 & $3.57 \times 10^{-13}$ \\
\hline CIL1338S0009 & Protein TIFY 6B & JAZ & TIFY6B & -4.081 & $4.52 \times 10^{-18}$ \\
\hline CIL1390S0024 & Protein TIFY 6B & JAZ & TIFY6B & -5.604 & $1.59 \times 10^{-14}$ \\
\hline CIL1565S0018 & Protein TIFY 6B & JAZ & TIFY6B & -3.637 & $1.25 \times 10^{-12}$ \\
\hline MSTRG.9204 & Protein TIFY 10A & JAZ & - & -3.812 & 0.016461 \\
\hline CIL1399S0034 & Transcription factor MYC2 & MYC2 & BHLH14 & -3.273 & $9.07 \times 10^{-10}$ \\
\hline MSTRG.9232 & Transcription factor MYC4 & MYC2 & MYC4 & 6.314 & $2 \times 10^{-22}$ \\
\hline \multicolumn{6}{|c|}{ SA } \\
\hline CIL1326S0021 & Transcription factor TGA7-like & TGA & TGA7 & 1.407 & $1.05 \times 10^{-15}$ \\
\hline CIL0037S0002 & Pathogenesis-related protein 1 & PR-1 & PRB1 & 8.827 & $1.24 \times 10^{-60}$ \\
\hline CIL0037S0003 & Pathogenesis-related protein 1 & PR-1 & PRB1 & 3.015 & $1.31 \times 10^{-16}$ \\
\hline CIL0037S0006 & Pathogenesis-related protein 1 & PR-1 & At2g14610 & 8.831 & $1.4 \times 10^{-139}$ \\
\hline CIL0037S0007 & Basic form of pathogenesis-related protein 1 & PR-1 & PRMS & 10.196 & $6.07 \times 10^{-5}$ \\
\hline CIL0232S0001 & Pathogenesis-related protein 1 & PR-1 & PRB1 & -4.242 & $9.5 \times 10^{-24}$ \\
\hline
\end{tabular}

3.5. Key Identified Differentially Expressed Proteins Involved in Starch and Sucrose Metabolism and Galactose Metabolism

Our analysis reveals that the transcriptional activity of genes involved in starch and sucrose metabolism and galactose metabolism were significantly regulated in the abscised fruitlets of pecan (Table 4 and Supplementary Table S4). The differential expression of transcripts coding for enzymes involved in sucrose, glucose, trehalose, starch, galactose, and galactinol metabolism was analyzed. For sucrose metabolism, the expression of two sucrose phosphate synthase (SPS) genes (EC: 2.4.1.14) were downregulated and CWINV1 (invertase, EC: 3.2.1.26) expression was upregulated in abscised fruitlets of pecan. All 4-glucan endo-1,3-beta-glucosidase genes (EC: 3.2.1.39), 6 out of 10 detected genes coding $\beta$ glucosidases (EC: 3.2.1.21), and an endoglucanase gene (EC: 3.2.1.4) for glucose metabolism were downregulated in abscised fruitlets compared with persisting fruitlets. For starch metabolism, two granule-bound starch synthase 1 (GBSS1, EC: 2.4.1.242), one GBSS2, and two 1,4-a-glucan branching enzyme (GBE, EC: 2.4.1.18) genes were downregulated in abscised fruitlets; however, two starch degradation alpha-amylase (EC: 3.2.1.1) genes were upregulated. For trehalose metabolism, the expression of trehalose 6-phosphate synthase (TPS, EC: 2.4.1.25) and trehalose-6-phosphate phosphatase (TPP, EC: 3.1.3.12) was higher in abscised fruitlets than in persisting fruitlets. The expression of alpha-galactosidase gene (EC: 3.2.1.22) was downregulated, but the expression of aldose 1-epimerase gene (EC: 5.1.3.3) was enhanced for galactose synthesis metabolism. Three out of 4 inositol 3-alphagalactosyltransferases (GolS, EC: 4.1.123) were downregulated in abscised pecan fruitlets.

Table 4. Response of transcription factors in comparison of A1 vs. P1.

\begin{tabular}{cccc}
\hline Category & Total & Upregulated & Downregulated \\
\hline MYB & 40 & 10 & 30 \\
NAC & 24 & 13 & 11 \\
bHLH & 24 & 4 & 20 \\
WRKY & 24 & 17 & 7 \\
bZIP & 4 & 1 & 3 \\
C2 & 2 & 0 & 2 \\
C2H2 & 1 & 0 & 1 \\
MADS & 3 & 0 & 3 \\
ERF & 25 & 11 & 14 \\
AUX/IAA & 25 & 4 & 21 \\
zinc finger & 69 & 15 & 54 \\
B3 & 4 & 1 & 4 \\
\hline
\end{tabular}




\subsection{Plant Hormone Content Analysis}

The contents of plant hormones 3-indolebutyric acid (IBA), ABA, GA, and Transzeatin riboside (TZR) were measured in abscised and persisting fruitlets at 35 and 48 DAA to further understand changes in plant hormone content after fruit shedding in pecan (Figure 4). As the fruit developed, the IBA content increased significantly, but ABA and GA content decreased significantly, indicating that increased IBA content and decreased ABA and GA might promote fruit development in pecan. Compared with persisting fruitlets, the IBA content was not changed in abscised fruitlets at 35 DAA but decreased significantly at 48 DAA. The ABA content was significantly lower in abscised fruitlets than in persisting fruitlets at 36 DAA, but the opposite was the case at 48 DAA. The GA content was significantly higher in abscised fruitlets than in persisting fruitlets, and the TZR content was significantly lower in abscised fruitlets than in persisting fruitlets at 36 and 48 DAA.

IBA

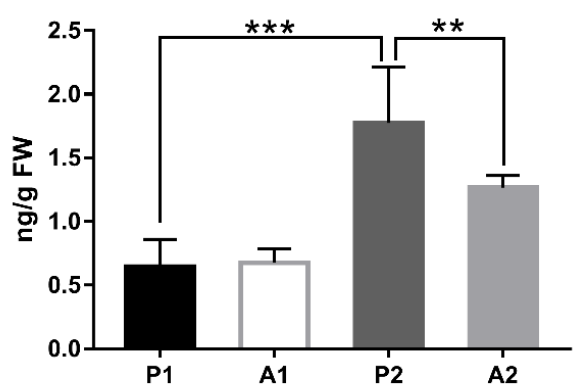

GA

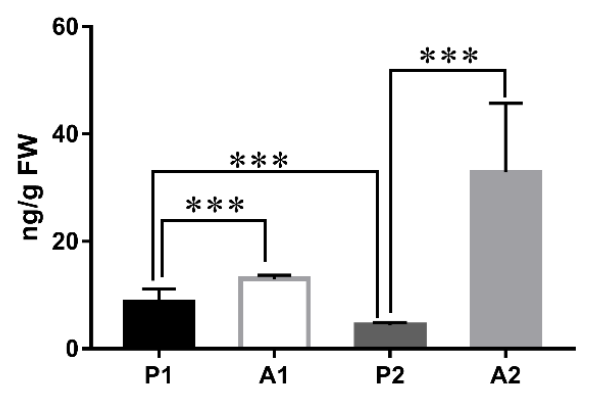

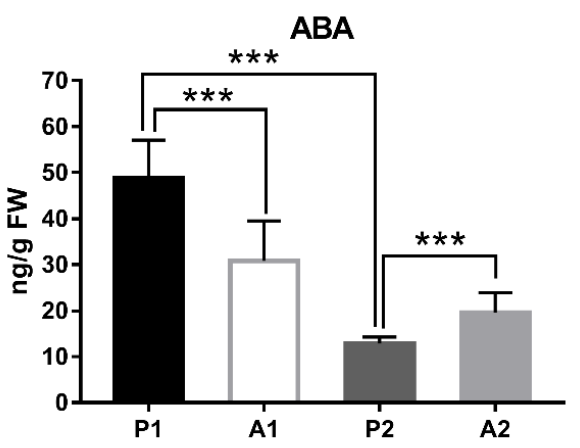

TZR

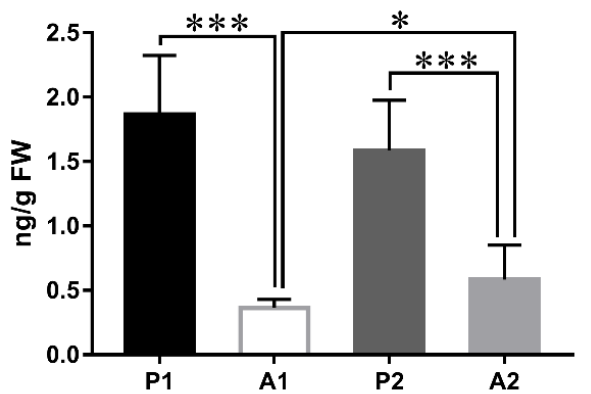

Figure 4. Analysis of plant hormone content in abscised and persisting fruitlets at 35 and 48 DAA. P1, persisting fruitlets at $35 \mathrm{DAA}$; 2 , persisting fruitlets at $48 \mathrm{DAA}$; A1, abscised fruitlets at $35 \mathrm{DAA}$; 2 , abscised fruitlets at 48 DAA. Statistically significant differences were calculated with GraphPad Prism 7.00. Data represent average \pm SD of three biological repeats with three measurements. * Significant differences at $p<0.05 ;{ }^{* *}$ significant differences at $p<0.01 ;{ }^{* * *}$ significant differences at $p<0.001$.

\subsection{Response of Transcription Factors in the Comparison of A1 vs. P1}

Differential expression of transcription factor genes was analyzed to identify transcription factors involved in abscised fruitlets in pecan (Table 4 and Supplementary Table S9). We identified 40 MYB transcription factors with significantly differential expression, 10 upregulated and 30 downregulated, suggesting that these factors might be involved in abscising in pecan. Among the NAM, ATAF1-2, and cup-shaped cotyledon 2 (NAC) transcription factor family, 13 members were upregulated and 11 members were downregulated in abscised compared with persisting fruitlets. WRKY, basic helix-loop-helix protein (bHLH), basic region/ leucine zipper motif (bZIP), C2C2, C2, ethylene response factor (ERF), zinc finger, and B3 transcription factor families were up- or downregulated in abscised fruitlets vs. persisting fruitlets, suggesting that these families may also play key roles in the transcriptional regulation of genes in abscission in the early stage of fruit development in pecan. 


\section{Discussion}

\subsection{Increased IBA Content and Decreased ABA and GA Might Promote Fruit Development in Pecan}

Previous reports showed that the ethylene and ABA molecular biosynthesis and action mechanisms appear to be significantly downregulated as soon as fruit set is triggered, and concomitantly, auxin and GA molecular biosynthesis and action mechanisms are activated $[10,11]$, indicating that fruit development appears to rely on the removal of a negative feedback regulation established by a negative control exerted mainly by ABA- and ethylene-dependent pathways of ovary growth [11]. Auxins may trigger cell division, and their interaction with GAs may be essential for sustaining cell expansion [9]. In this study, the IBA content was increased significantly, but the ABA and GA contents were decreased significantly as the fruit developed at the early stage (Figure 4), indicating that increased IBA and decreased ABA and GA might promote fruit development in pecan. Thus, further studies are needed on the molecular role of GA in the development of pecan.

\subsection{Plant Hormones Seem to Play a Key Role during the Abscission Progress in Pecan}

The IBA content of abscised fruitlets did not change abruptly at 35 DAA compared with persisting fruitlets, but decreased significantly at 48 DAA; however, the ABA content decreased significantly at 35 DAA but increased abruptly at 48 DAA, and the GA content was significantly higher in abscised fruitlets than in persisting fruitlets. These results show that the changes in plant hormones in abscised and persisting fruitlets have different trends at different stages of development in pecan. At 42 DAA, increased ABA and GA and decreased IBA and TZR might promote fruitlet abscission in pecan, but downregulation of GA signaling specifically in fruits induced to abscise in apple [5]. At 35 DAA, decreased ABA and no change in IAA were observed by comparing their contents in abscised and persisting fruitlets. Further studies are needed to study the initiation of abscission in pecan regarding plant hormone signaling, especially GAs, at the early stage.

Our transcriptomic data suggest that the majority of transcriptionally activated genes involved in hormone signaling were downregulated in abscised fruitlets, except for ethylene and salicylic acid signaling (Table 3 and Supplementary Table S8), indicating that hormones may play an important role during the progress of abscission in pecan. Botton et al. reported that $\mathrm{ABA}$ and ethylene signaling were strongly upregulated concurrently, with downregulation of GA signaling specifically in fruits induced to abscise in apple [5]. The essential role of ABA in abscission has been broadly studied in different species [4]. Exogenous ABA treatment can induce fruitlet abscission in the apple L3 class, and ABA may be involved in upstream induction of abscission [4]. Increased levels of ABA observed in BA-treated apple L3 fruitlets that were abscising, concurrently with upregulation of the $A B A$-responsive 9-cis-epoxycarotenoid dioxygenase 1 (MdNCED1) gene [26], suggest activation of the indirect biosynthetic pathway of ABA [4]. In our study, two NCED encoding genes were downregulated in abscised pecan fruitlets (Supplementary Table S8), consistent with the decreased ABA content (Figure 4). In addition, 8 ABA signal transduction genes were downregulated in abscised pecan fruitlets. These results show that ABA signaling is strongly downregulated in abscised pecan fruitlets, but on the contrary, it is strongly upregulated in apple fruits induced to abscise [5].

Ethylene signaling was significantly enhanced in abscised pecan fruitlets, as shown by high expression of some ethylene signaling genes (Table 3). Mitogen-activated protein kinase $(M A P K)$ genes, which were found to be upregulated in abscised fruitlets (Supplementary Table S8), are key elements of the ethylene signal transduction pathway, probably involved in ethylene-ABA crosstalk [27]. The related proteins (14-3-3 genes), which were also found to be upregulated in abscised fruitlets (Supplementary Table S8), may trigger ABAethylene crosstalk and the response to sugar starvation [28]. The TPS gene, found to be overexpressed in abscised fruitlets, may also regulate ABA signaling, as found in Arabidopsis [29] and apple [5]. ABA and ethylene signaling are strongly upregulated concurrently with downregulation of GA signaling specifically in apple fruits induced to 
abscise [5]. Our transcriptomic data show that ethylene signaling is strongly upregulated concurrently with downregulation of GA and ABA signaling in abscised pecan fruitlets.

Auxin participates in plant organ abscission. Transcript analysis revealed that auxin may regulate the expression of early auxin-responsive gene families including $A U X / I A A$, gretchen Hagen3 (GH3), and small auxin up RNA (SAUR). Auxin regulates the expression of various ARFs during early abscission, whereas ethylene has the opposite effect on most of these genes in tomato [30]. SlARFs have overlapping functions in the abscission process. Meir et al. reported that tomato flower abscission was associated with the expression level of $A U X / I A A$ genes [31]. GH3 genes are also involved in fruitlet or flower abscission. LcAUX/IAA1 and LcSAUR1 may play more important roles in abscission than LcGH3.1 in litchi, because $L c A U X / I A A 1$ and $L c S A U R 1$ were mostly expressed in AZ [32]. Tomato GH3 increased slightly after $8 \mathrm{~h}$ and maintained a low expression level during abscission, implying that it may be an effective negative regulator in IAA-induced abscission delay [33]. $S A U R$, an auxin-responsive gene, may serve as a marker of IAA level throughout the abscission process [33]. Two SAUR-like genes were found to be involved in shadinginduced abscission by transcriptomic analysis in apple [34]. Girdling plus defoliation treatment could induce LcSAUR1 expression in AZ and fruitlet, and significantly induce litchi fruitlet abscission [32]. SAUR36 has been reported to be involved in leaf senescence in Arabidopsis [35]. Overexpression of OsSAUR39 in rice (Oryza sativa) results in phenotypes that include reductions in lateral root development, yield, and shoot and root length, suggesting that OsSAUR39 acts as a negative regulator of auxin synthesis and transport [36]. Fourteen CitSAUR genes showed obvious changes during citrus fruitlet abscission, and CitSAUR06, CitSAUR08, CitSAUR44, CitSAUR61, and CitSAUR64 were more relevant because their expression patterns under IAA treatment exhibited an opposite trend to that during fruitlet drop [37]. In our study, all $8 A U X / I A A, 3 A R F$, and 2 out of $3 G H 3$ genes detected were downregulated, but 3 out of 4 SAUR genes were upregulated in abscised pecan fruitlets. These results show that the roles of GH3, ARF, AUX/INN, and SAUR in fruitlet abscission may vary considerably among plant species.

Beyond the GA, ABA, auxin, and ethylene signaling discussed above, cytokinin, jasmonate, salicylic acid, and brassinosteroid signaling are also involved in abscised pecan fruitlets. An association of response variables with expression data of genes regarding cytokinin, jasmonates, salicylic acid, and brassinosteroids was detected in abscising apple fruitlets [5]. Especially related to salicylic acid, many genes related to metabolism were highly expressed in abscised fruitlets. A TGA gene and $4 P R-1$ genes were upregulated in abscised fruitlets, indicating that systemic acquired resistance (SAR) may be triggered in abscised pecan fruitlets.

\subsection{Reduced Sugar Supply in Abscised Fruitlets Is One Reason for Fruitlet Abscission in Pecan}

Sugar signaling is known to play a role in senescence regulation in a complex network. Thus, the senescence process is triggered in fruitlets once the sugar supply is decreased [38]. In apple, the sorbitol concentration of central fruitlets is higher than that of L1 pedicels, indicating that the former profit from a better supply of sugars. The increased supply of sugar to the central fruit would allow it to develop at a faster rate, thus further increasing its sink strength [6]. In our study, the single fruit mass of abscised fruitlets (just shed from the main plant body) was about half that of persisting fruitlets (Figure 1 and Supplementary Figure S1), indicating that abscised fruitlets had stopped growing and developing for some time before dropping, thus further indicating that the sugar supply is reduced or stopped in abscising pecan fruitlet.

The transcriptome analysis showed that genes coded for carbohydrate metabolism, including starch and sucrose, galactose, pyruvate, and amino sugar and nucleotide sugar metabolism, are significantly regulated in abscised pecan fruitlets (Table 5). Decreased expression of transcripts coding for enzymes involved in sucrose, glucose, and starch metabolism may be expected in slow-growing organs, considering that fruitlets act as a major carbon sink. SPS catalyzing fructose-6-phophate and UDP-glucose to synthesis sucrose-6-phosphate in plants is a key regulatory step in the control of sucrose synthesis [39]. Overexpression of SPS in Arabidopsis thaliana results in increased foliar sucrose/starch ratios 
and decreased foliar carbohydrate accumulation in plants after prolonged growth with $\mathrm{CO}_{2}$ enrichment [40]. Transformed tobacco plants overexpressing Arabidopsis SPS gene showed elevated transcript abundance and SPS enzyme activity, substantial pooling of soluble stem sucrose content, significantly increased stem height and greater stem diameters, longer fibers, and increased total dry biomass relative to control plants [41]. Vacuolar invertase (VINV) and cell wall invertase (CWINV, EC: 3.2.1.26) are invertases, cleaving sucrose to glucose and fructose. The transcripts and activities of CWINV, CytINV, and $V I N V$ at the fully mature stage were higher in Sweet Miriam, in agreement with the low sucrose content [42]. In our study, the expression of two SPS genes was downregulated and CWINV1 expression was upregulated in abscised fruitlets in pecan, suggesting that sucrose synthesis was reduced.

Plant class I glucan endo-1,3- $\beta$-glucosidases ( $\beta$-1,3-glucanase; 1,3 - $\beta$-D-glucan glucanohydrolase, EC: 3.2.1.39) have been implicated in development and the defense against pathogen attack. $\beta$-glucosidases (EC: 3.2.1.21) are glycosyl hydrolases that hydrolyze the $\beta$-O-glycosidic bond at the anomeric carbon of a glucose moiety at the nonreducing end of a carbohydrate or glycoside molecule [43]. Endoglucanases (EC: 3.2.1.4) are associated with fruit ripening, growth of cultured cells, and leaf abscission. In our study, all 4 glucan endo1,3- $\beta$-glucosidase genes, 6 out of 10 detected $\beta$-glucosidase genes, and a endoglucanase gene were downregulated in abscised fruitlets compared with persisting fruitlets, indicating that the glucose metabolism is blocked in abscised fruitlets in pecan.

Starch is the major storage carbohydrate in most plants, with many important functions. Granule-bound starch synthase (GBSS, EC: 2.4.1.242) is the glucosyl transferase specifically responsible for elongating amylose polymers and the only protein known to be required for its biosynthesis. Protein targeting of starch is required for localizing granule-bound starch synthase to starch granules and for normal amylose synthesis in Arabidopsis [44,45]. Two GBSS1 genes and one GBSS2 gene were downregulated in abscised fruitlets in pecan, indicating that starch biosynthesis was blocked. The 1,4- $\alpha$-glucan branching enzyme (GBE, EC: 2.4.1.18) is known to cleave the $\alpha-1,4$ glucosidic linkage of an existing glucan chain and transfer the cut end to the 6-position of a glucose residue within the cleaved chain or within another glucan chain, creating an $\alpha-1,6$ glucosidic linkage, increasing the ratio of amylopectin to amylose [46]. Amylopectin has a slower recrystallization rate than amylose. GBE treatment could retard both short- and long-term retrogradation of starch in corn [47]. It is well known that a-amylases are important enzymes for starch degradation in plants [48]. In this study, two GBE genes were downregulated and two starch degradation alpha-amylase genes were upregulated in abscised fruitlets in pecan, showing that starch retrogradation and degradation are intensified. These results show that starch biosynthesis was blocked and starch retrogradation and degradation were intensified, resulting in reduced starch content in pecan.

Trehalose is an effective signaling molecule that has been shown to function in carbohydrate storage [49]. The trehalose biosynthetic pathway is used to transfer Glc from UDP-Glc to Glc-6- $P$, resulting in trehalose-6- $P$ and UDP in plants. This initial step is catalyzed by TPS (EC: 2.4.1.15), and the dephosphorylation of trehalose-6-P occurs via TPP (EC: 3.1.3.12) producing trehalose in a second step [50]. Arabidopsis class I genes (AtTPS1-4) regulate starch storage, resistance to drought, and inflorescence architecture. Class II genes (AtTPS5-11) encode multifunctional enzymes with synthase and phosphatase activity [49], induced by sugar starvation [51]. Trehalose-6-phosphate, which is a sugar signal, induces a prompt reaction to nutritional stress during the early induction of abscission. The expression levels of a class II TPS gene were found to be high in the cortex of abscising fruitlets of apple and citrus [5]. Celton et al. reported that TPP and TPS genes showed increased expression in the pedicels of apple central fruitlets, which were persisting fruits, indicating that central pedicels may be supplied from a very early stage of development in apple [6]. Overexpression of Escherichia coli TPS (OTS A) in transgenic tobacco was shown to increase photosynthetic activity, but constitutive expression of TPP (OTS B) resulted in reduced photosynthesis [52]. However, the expression of TPS and TPP in was higher in abscised fruitlets than in persisting fruitlets in 
pecan in our study, indicating that the functions of trehalose may vary among plants, which needs further validation in the early stage of pecan fruitlet abscission.

Table 5. Starch and sucrose metabolism and galactose metabolism.

\begin{tabular}{|c|c|c|c|c|c|}
\hline Gene ID & Description & EC No. & Symbol & $\log 2(f c)$ & $p$-Value \\
\hline CIL1395S0001 & Alpha-glucosidase & 3.2 .1 .20 & - & 1.594 & 0.012 \\
\hline MSTRG.2545 & Alpha-glucosidase & 3.2.1.20 & Os06g0675700 & 1.429 & $2.71 \times 10^{-8}$ \\
\hline CIL1595S0049 & Glucose-1-phosphate adenylyltransferase & 2.7.7.27 & $\operatorname{glg} C$ & -2.746 & $2.81 \times 10^{-16}$ \\
\hline CIL0203S0004 & Glucose-1-phosphate adenylyltransferase & 2.7.7.27 & $\operatorname{glg} C$ & -3.281 & $1.24 \times 10^{-10}$ \\
\hline MSTRG.12720 & Alpha-amylase-like isoform $\mathrm{X} 1$ & 3.2.1.1 & AMY1.1 & 3.719 & $1.74 \times 10^{-76}$ \\
\hline MSTRG.2528 & Alpha-amylase-like isoform $\mathrm{X} 1$ & 3.2.1.1 & AMY1.1 & 1.358 & $6.01 \times 10^{-13}$ \\
\hline CIL1383S0034 & Glucan endo-1,3-beta-glucosidase 1 & 3.2.1.39 & At1g11820 & -3.735 & $1.49 \times 10^{-32}$ \\
\hline CIL1482S0014 & Glucan endo-1,3-beta-glucosidase 4-like & 3.2.1.39 & At3g13560 & -3.409 & $2.95 \times 10^{-15}$ \\
\hline CIL1332S0070 & Glucan endo-1,3-beta-glucosidase 5-like & 3.2.1.39 & At4g31140 & -1.632 & $4.40 \times 10^{-4}$ \\
\hline CIL1347S0008 & Glucan endo-1,3-beta-glucosidase 6 & 3.2.1.39 & At5g58090 & -6.006 & $5.41 \times 10^{-32}$ \\
\hline CIL1359S0017 & Hexokinase-3-like [Juglans regia] & 2.7.1.1 & At1g50460 & -1.211 & $3.43 \times 10^{-3}$ \\
\hline MSTRG.14644 & Hexokinase-3-like isoform X3 & 2.7.1.1 & At1g50460 & -2.125 & $2.03 \times 10^{-7}$ \\
\hline CIL1459S0007 & Hexokinase-3-like isoform X2 & 2.7.1.1 & At1g50460 & -2.411 & $5.93 \times 10^{-7}$ \\
\hline CIL0282S0003 & Hexokinase-1-like isoform X2 & 2.7.1.1 & HXK1 & 1.958 & $1.53 \times 10^{-12}$ \\
\hline CIL1518S0008 & Hexokinase-2 & 2.7.1.1 & HXK2 & -2.598 & 0.005 \\
\hline CIL1568S0010 & Probable fructokinase-7 & 2.7.1.4 & At5g51830 & 2.623 & $2.65 \times 10^{-36}$ \\
\hline CIL0508S0004 & Beta-glucosidase 12-like isoform X3 & 3.2.1.21 & BGLU12 & -3.745 & 0.003 \\
\hline CIL1537S0001 & Beta-glucosidase 12-like & 3.2.1.21 & BGLU12 & -8.471 & $5.16 \times 10^{-51}$ \\
\hline CIL0493S0002 & Beta-glucosidase 12-like & 3.2.1.21 & BGLU13 & 11.919 & $2.12 \times 10^{-11}$ \\
\hline MSTRG.6474 & Beta-glucosidase 12-like & 3.2.1.21 & BGLU13 & 10.737 & $1.03 \times 10^{-7}$ \\
\hline CIL0508S0002 & Beta-glucosidase 13-like isoform X2 & 3.2.1.21 & BGLU13 & -2.922 & $1.26 \times 10^{-4}$ \\
\hline MSTRG.7161 & Beta-glucosidase 12-like & 3.2.1.21 & BGLU24 & -9.010 & $1.78 \times 10^{-54}$ \\
\hline CIL1405S0071 & Beta glucosidase 41 isoform 2 & 3.2.1.21 & BGLU25 & -3.444 & $1.12 \times 10^{-11}$ \\
\hline CIL0391S0004 & Beta-glucosidase 42 isoform $\mathrm{X} 1$ & 3.2.1.21 & BGLU42 & -4.571 & $2.02 \times 10^{-52}$ \\
\hline CIL1320S0039 & Beta-glucosidase 47 -like isoform $\mathrm{X} 1$ & 3.2.1.21 & BGLU47 & 4.020 & $9.97 \times 10^{-18}$ \\
\hline CIL1407S0038 & Endoglucanase 8-like & 3.2.1.4 & CEL1 & -6.822 & $8.06 \times 10^{-27}$ \\
\hline MSTRG.23283 & Beta-glucosidase & 3.2.1.21 & RE1 & 2.092 & $1.75 \times 10^{-3}$ \\
\hline CIL1317S0076 & Beta-fructofuranosidase & 3.2.1.26 & CWINV1 & 4.017 & $8.53 \times 10^{-14}$ \\
\hline CIL1506S0011 & Beta-fructofuranosidase & 3.2.1.26 & CWINV3 & -5.878 & $1.01 \times 10^{-4}$ \\
\hline CIL1264S0043 & Acid beta-fructofuranosidase-like & 3.2.1.26 & $\mathrm{INV}^{*} \mathrm{DC} 4$ & -3.426 & $1.78 \times 10^{-25}$ \\
\hline CIL0525S0001 & Nudix hydrolase 14 , chloroplastic & 3.6.1.21 & NUDT14 & -1.662 & $8.32 \times 10^{-4}$ \\
\hline CIL1568S0006 & Phosphoglucomutase, chloroplastic & 5.4 .2 .2 & PGMP & -1.205 & 0.011 \\
\hline CIL0360S0002 & Sucrose-phosphate synthase 1 & 2.4.1.14 & SPS1 & -1.765 & $8.57 \times 10^{-3}$ \\
\hline CIL1271S0008 & Sucrose-phosphate synthase 1 & 2.4.1.14 & SPS1 & -7.197 & $1.16 \times 10^{-29}$ \\
\hline CIL1417S0045 & 1,4-alpha-glucan-branching enzyme 3 & 2.4.1.18 & GBE3, glgB, SBE3 & -4.466 & $5.87 \times 10^{-16}$ \\
\hline CIL1531S0004 & 1,4-alpha-glucan-branching enzyme 1 & 2.4.1.18 & GBE3, glgB, SBEI & -2.321 & $4.31 \times 10^{-18}$ \\
\hline CIL0218S0017 & Granule-bound starch synthase 1 & 2.4.1.242 & WAXY, GBSS1 & -3.748 & $8.21 \times 10^{-14}$ \\
\hline CIL0389S0009 & Granule-bound starch synthase 1 & 2.4.1.242 & WAXY, GBSS1 & -12.135 & $6.74 \times 10^{-12}$ \\
\hline CIL0176S0049 & Granule-bound starch synthase 2 & 2.4.1.21 & SS2 & -5.083 & $1.69 \times 10^{-20}$ \\
\hline CIL1531S0021 & Trehalose-phosphate phosphatase & 3.1.3.12 & TPP & 1.896 & $3.70 \times 10^{-5}$ \\
\hline CIL1310S0034 & Trehalose 6-phosphate synthase & 2.4.1.15 & TPS & 1.535 & $1.66 \times 10^{-12}$ \\
\hline CIL0021S0018 & Alpha-galactosidase 1-like & 3.2.1.22 & AGAL1 & -2.014 & $3.88 \times 10^{-12}$ \\
\hline CIL1429S0021 & Aldose 1-epimerase & 5.1.3.3 & Galm & 1.077 & $4.95 \times 10^{-48}$ \\
\hline CIL0309S0003 & Inositol 3-alpha-galactosyltransferase 1 & 2.4.1.123 & GOLS1 & 1.063 & $1.44 \times 10^{-11}$ \\
\hline MSTRG.21476 & Inositol 3-alpha-galactosyltransferase 1 & 2.4.1.123 & GOLS1 & -13.936 & $3.70 \times 10^{-15}$ \\
\hline MSTRG.20754 & Inositol 3-alpha-galactosyltransferase 2 & 2.4.1.123 & GOLS2 & -2.722 & 0.013 \\
\hline MSTRG.20755 & Inositol 3-alpha-galactosyltransferase 2 & 2.4.1.123 & GOLS2 & -2.837 & 0.021 \\
\hline CIL0344S0021 & 6-phosphofructokinase 1 & 2.7.1.1 & PFK3 & -4.090 & $2.05 \times 10^{-8}$ \\
\hline CIL1568S0006 & Phosphoglucomutase & 5.4.2.2 & PGMP & -1.205 & 0.011 \\
\hline CIL1358S0005 & Raffinose synthase & 2.4.1.82 & RFS6 & 3.211 & $2.01 \times 10^{-33}$ \\
\hline CIL0272S0009 & UDP-glucose 4-epimerase GEPI48-like & 5.1 .3 .2 & UGE5 & 1.349 & $1.70 \times 10^{-11}$ \\
\hline CIL1564S0017 & UDP-glucose 4-epimerase GEPI48 & 5.1.3.2 & UGE5 & 3.159 & $8.72 \times 10^{-43}$ \\
\hline CIL1297S0013 & UDP-sugar pyrophosphorylase & 2.7.7.64 & USP & -3.752 & $4.18 \times 10^{-8}$ \\
\hline
\end{tabular}


Previous reports showed that galactose synthesis via alpha-galactosidase (EC: 3.2.1.22) results from the hydrolysis of raffinose to yield free galactose and sucrose [53]. $\alpha-G A L$ and $\beta-G A L$ transcripts were higher in Santa Rosa fruits and leaves than in Sweet Miriam and correlated well with the higher Santa Rosa galactose content [42]. In our study, the expression of gene coding alpha-galactosidase was downregulated, but the expression of gene coding aldose 1-epimerase (EC: 5.1.3.3) was enhanced, indicating that galactose synthesis is reduced and degradation is enhanced in abscised fruitlets. UDP-Gal together with myo-inositol are used as substrates by GolS(EC: 4.1.123) for the synthesis of galactinol [54]. Three out of 4 genes coding GolS were downregulated in abscised pecan fruitlets, showing that galactinol synthesis was also blocked.

\subsection{NAC Transcription Factors Participate in Fruit Development or Abscission Process}

Multiple NAC family proteins were identified in abscised fruitlets in our study. The NAC proteins, which constitute one of the largest major transcription factor families, are well-known for their roles in several developmental programs [55]. NAC TFs have been shown to play important roles in various biological processes, as well as responses to abiotic stresses, and are important regulators in a wide range of developmental processes, such as the formation of lateral roots, the development of shoot apical meristem, floral morphogenesis, embryo development, grain nutrient remobilization, and cell wall biosynthesis [56]. In kiwifruit, 74 of 142 NACs were found to be persistently expressed in fruit during the whole developmental process, and crucial candidate NAC genes were shown to be involved in fruit growth and development [57]. Several NAC transcription factors were upregulated in the pedicels of central fruits, which were persisting fruits, in Malus domestica, which might contribute to increased vascular development in the central pedicel in the early developmental stages [6]. In our study, 24 NAC transcription factors were upor downregulated in abscised fruitlets, indicating that these genes participate in the fruit development or abscission process.

\section{Conclusions}

The fruit development and drop dynamics of the pecan 'Pawnee' were studied in this paper, and the results show that the fruit drop rate was more than $57 \%$, suggesting that fruit drop was very serious. In order to understand the mechanism of fruitlet drop, transcriptomic profiling of persisting and abscised fruitlets in early fruit development was conducted by RNA-seq. A total of 11,976 DEGs were identified, 3012 upregulated and 8964 downregulated, in the comparison of abscised vs. persisting fruitlets at 35 DAA. Our transcriptomic data suggest that a majority of the transcriptionally activated genes involved in hormone signaling were downregulated in the abscised fruitlets, except for ethylene and salicylic acid signaling. Unlike the downregulation of GA signaling specifically in fruits induced to abscise in apple [5], the GA content was increased in abscised fruitlets in pecan. Thus, the involvement of plant hormone signaling, especially GAs, at the early stage of fruit abscission should be studied in the future. Transcripts coding for enzymes involved in sucrose, glucose, trehalose, starch, galactose, and galactinol metabolism were reduced, suggesting that abscised pecan fruitlets stopped growing and developing for some time before dropping, indicating that their sugar supply was reduced or stopped. The transcriptome characterization described in this paper contributes to unravelling the molecular mechanisms and pathways involved in the physiological abscission of pecan fruits.

Supplementary Materials: The following are available online at https:/ / www.mdpi.com/article/10 .3390/cimb44010013/s1.

Author Contributions: J.Z. and G.W. designed the experiments and wrote the manuscript; J.Z., F.Z., T.W., Y.L. and G.W. performed the experiments; J.Z. and F.Z. analyzed the data. All authors have read and agreed to the published version of the manuscript. 
Funding: This research was funded by the Independent Innovation of Agricultural Science and Technology of Jiangsu Province (grant number CX(21)3046), the Science and Technology Plan Project of Nanjing (grant number 20201103), and the Science and Technology Plan Project of Luhe District of Nanjing (grant number LHZC2021N01).

Institutional Review Board Statement: Not applicable.

Informed Consent Statement: Not applicable.

Data Availability Statement: The data presented in this study are openly available in NCBI SRA (http:/ / www.ncbi.nlm.nih.gov/sra), reference number [PRJNA784729].

Acknowledgments: Not applicable.

Conflicts of Interest: The authors declare no conflict of interest.

\section{References}

1. Estornell, L.H.; Agusti, J.; Merelo, P.; Talon, M.; Tadeo, F.R. Elucidating mechanisms underlying organ abscission. Plant Sci. 2013, 199-200, 48-60. [CrossRef]

2. Patharkar, O.R.; Walker, J.C. Advances in abscission signaling. J. Exp. Bot. 2018, 69, 733-740. [CrossRef]

3. Eccher, G.; Begheldo, M.; Boschetti, A.; Ruperti, B.; Botton, A. Roles of ethylene production and ethylene receptor expression in regulating apple fruitlet abscission. Plant Physiol. 2015, 169, 125-137. [CrossRef]

4. Giulia, E.; Alessandro, B.; Mariano, D.; Andrea, B.; Benedetto, R.; Angelo, R. Early induction of apple fruitlet abscission is characterized by an increase of both isoprene emission and abscisic acid content. Plant Physiol. 2013, 161, 1952-1969. [CrossRef]

5. Botton, A.; Eccher, G.; Forcato, C.; Ferrarini, A.; Begheldo, M.; Zermiani, M.; Moscatello, S.; Battistelli, A.; Velasco, R.; Ramina, R.A.J.P.P. Signaling pathways mediating the induction of apple fruitlet abscission. Plant Physiol. 2011, 155, 185-208. [CrossRef]

6. Celton, J.-M.; Dheilly, E.; Guillou, M.-C.; Simonneau, F.; Juchaux, M.; Costes, E.; Laurens, F.; Renou, J.-P. Additional amphivasal bundles in pedicel pith exacerbate central fruit dominance and induce self-thinning of lateral fruitlets in apple. Plant Physiol. 2014, 164, 1930-1951. [CrossRef]

7. Sundaresan, S.; Philosoph-Hadas, S.; Riov, J.; Mugasimangalam, R.; Kuravadi, N.A.; Kochanek, B.; Salim, S.; Tucker, M.L.; Meir, S. De novo transcriptome sequencing and development of abscission zone-specific microarray as a new molecular tool for analysis of tomato organ abscission. Front. Plant Sci. 2015, 6, 1258. [CrossRef]

8. Sundaresan, S.; Philosoph-Hadas, S.; Ma, C.; Jiang, C.Z.; Riov, J.; Mugasimangalam, R.; Kochanek, B.; Salim, S.; Reid, M.S.; Meir, S. The tomato hybrid proline-rich protein regulates the abscission zone competence to respond to ethylene signals. Hortic. Res. 2018, 5, 28. [CrossRef]

9. de Jong, M.; Mariani, C.; Vriezen, W.H. The role of auxin and gibberellin in tomato fruit set. J. Exp. Bot. 2009, 60, 1523-1532. [CrossRef]

10. Nitsch, L.M.; Oplaat, C.; Feron, R.; Ma, Q.; Wolters-Arts, M.; Hedden, P.; Mariani, C.; Vriezen, W.H. Abscisic acid levels in tomato ovaries are regulated by LeNCED1 and SICYP707A1. Planta 2009, 229, 1335-1346. [CrossRef]

11. Vriezen, W.H.; Feron, R.; Maretto, F.; Keijman, J.; Mariani, C. Changes in tomato ovary transcriptome demonstrate complex hormonal regulation of fruit set. New Phytol. 2008, 177, 60-76. [CrossRef]

12. Cin, V.D.; Danesin, M.; Boschetti, A.; Dorigoni, A.; Ramina, A. Ethylene biosynthesis and perception in apple fruitlet abscission (Malus domestica L. Borck). J. Exp. Bot. 2005, 56, 2995-3005. [CrossRef]

13. Kuhn, N.; Serrano, A.; Abello, C.; Arce, A.; Espinoza, C.; Gouthu, S.; Deluc, L.; Arce-Johnson, P. Regulation of polar auxin transport in grapevine fruitlets (Vitis vinifera L.) and the proposed role of auxin homeostasis during fruit abscission. BMC Plant Biol. 2016, 16, 234. [CrossRef]

14. Rook, F.; Corke, F.; Baier, M.; Holman, R.; May, A.G.; Bevan, M.W. Impaired sucrose induction1 encodes a conserved plant-specific protein that couples carbohydrate availability to gene expression and plant growth. Plant J. 2006, 46, 1045-1058. [CrossRef]

15. Rorat, T. Plant dehydrins-tissue location, structure and function. Cell. Mol. Biol. Lett. 2006, 11, 536-556. [CrossRef]

16. Herrera-Rodriguez, M.B.; Maldonado, J.M.; Perez-Vicente, R. Light and metabolic regulation of HAS1, HAS1.1 and HAS2, three asparagine synthetase genes in Helianthus annuus. Plant Physiol. Biochem. 2004, 42, 511-518. [CrossRef]

17. Grauke, L.J.; Wood, B.W.; Harris, M.K. Crop Vulnerability: Carya. HortScience 2016, 51, 653-663. [CrossRef]

18. Poletto, T.; Muniz, M.F.B.; Poletto, I.; Baggiotto, C. Methods for overcome dormancy of pecan carya illinoinensis (wangenh.) k. koch seeds. Rev. Árvore 2015, 39, 1111-1118. [CrossRef]

19. Chen, S.; Zhou, Y.; Chen, Y.; Jia, G. Fastp: An ultra-fast all-in-one FASTQ preprocessor. Bioinformatics 2018, 34, i884-i890. [CrossRef]

20. Langmead, B.; Salzberg, S.L. Fast gapped-read alignment with Bowtie 2. Nat. Methods 2012, 9, 357-359. [CrossRef]

21. Kim, D.; Langmead, B.; Salzberg, S.L. HISAT: A fast spliced aligner with low memory requirements. Nat. Methods 2015, 12, 357-360. [CrossRef]

22. Pertea, M.; Pertea, G.M.; Antonescu, C.M.; Chang, T.C.; Mendell, J.T.; Salzberg, S.L. StringTie enables improved reconstruction of a transcriptome from RNA-seq reads. Nat. Biotechnol. 2015, 33, 290-295. [CrossRef] 
23. Anders, S.; Pyl, P.T.; Huber, W. HTSeq-A Python framework to work with high-throughput sequencing data. Bioinformatics 2015, 31, 166-169. [CrossRef]

24. Trapnell, C.; Williams, B.A.; Pertea, G.; Mortazavi, A.; Kwan, G.; van Baren, M.J.; Salzberg, S.L.; Wold, B.J.; Pachter, L. Transcript assembly and quantification by RNA-Seq reveals unannotated transcripts and isoform switching during cell differentiation. Nat. Biotechnol. 2010, 28, 511-515. [CrossRef]

25. Love, M.I.; Huber, W.; Anders, S. Moderated estimation of fold change and dispersion for RNA-seq data with DESeq2. Genome Biol. 2014, 15, 550. [CrossRef]

26. Schwartz, S.H.; Qin, X.; Zeevaart, J.A. Elucidation of the indirect pathway of abscisic acid biosynthesis by mutants, genes, and enzymes. Plant Physiol 2003, 131, 1591-1601. [CrossRef]

27. Xin, Z.; Zhao, Y.; Zheng, Z.L. Transcriptome analysis reveals specific modulation of abscisic acid signaling by ROP10 small GTPase in Arabidopsis. Plant Physiol. 2005, 139, 1350-1365. [CrossRef]

28. Lancien, M.; Roberts, M.R. Regulation of Arabidopsis thaliana 14-3-3 gene expression by gamma-aminobutyric acid. Plant Cell Environ. 2006, 29, 1430-1436. [CrossRef]

29. Avonce, N.; Leyman, B.; Mascorro-Gallardo, J.O.; Van Dijck, P.; Thevelein, J.M.; Iturriaga, G. The Arabidopsis trehalose-6-P synthase AtTPS1 gene is a regulator of glucose, abscisic acid, and stress signaling. Plant Physiol. 2004, 136, 3649-3659. [CrossRef]

30. Guan, X.; Xu, T.; Gao, S.; Qi, M.; Wang, Y.; Liu, X.; Li, T. Temporal and spatial distribution of auxin response factor genes during tomato flower abscission. J. Plant Growth Regul. 2013, 33, 317-327. [CrossRef]

31. Meir, S.; Philosoph-Hadas, S.; Sundaresan, S.; Selvaraj, K.S.; Burd, S.; Ophir, R.; Kochanek, B.; Reid, M.S.; Jiang, C.Z.; Lers, A. Microarray analysis of the abscission-related transcriptome in the tomato flower abscission zone in response to auxin depletion Plant Physiol. 2010, 154, 1929-1956. [CrossRef]

32. Kuang, J.F.; Wu, J.Y.; Zhong, H.Y.; Li, C.Q.; Chen, J.Y.; Lu, W.J.; Li, J.G. Carbohydrate stress affecting fruitlet abscission and expression of genes related to auxin signal transduction pathway in litchi. Int. J. Mol. Sci. 2012, 13, 16084-16103. [CrossRef]

33. Zuo, X.; Xu, T.; Qi, M.; Lv, S.; Li, J.; Gao, S.; Li, T. Expression patterns of auxin-responsive genes during tomato flower pedicel abscission and potential effects of calcium. Aust. J. Bot. 2012, 60, 68. [CrossRef]

34. Zhu, H.; Dardick, C.D.; Beers, E.P.; Callanhan, A.M.; Xia, R.; Yuan, R. Transcriptomics of shading-induced and NAA-induced abscission in apple (Malus domestica) reveals a shared pathway involving reduced photosynthesis, alterations in carbohydrate transport and signaling and hormone crosstalk. BMC Plant Biol. 2011, 11, 138. [CrossRef]

35. Hou, K.; Wu, W.; Gan, S.S. SAUR36, a small auxin up RNA gene, is involved in the promotion of leaf senescence in Arabidopsis Plant Physiol. 2013, 161, 1002-1009. [CrossRef]

36. Kant, S.; Bi, Y.M.; Zhu, T.; Rothstein, S.J. SAUR39, a small auxin-up RNA gene, acts as a negative regulator of auxin synthesis and transport in rice. Plant Physiol. 2009, 151, 691-701. [CrossRef]

37. Xie, R.; Dong, C.; Ma, Y.; Deng, L.; He, S.; Yi, S.; Lv, Q.; Zheng, Y. Comprehensive analysis of SAUR gene family in citrus and its transcriptional correlation with fruitlet drop from abscission zone A. Funct. Integr. Genom. 2015, 15, 729-740. [CrossRef]

38. Baena-Gonzalez, E.; Rolland, F.; Thevelein, J.M.; Sheen, J. A central integrator of transcription networks in plant stress and energy signalling. Nature 2007, 448, 938-942. [CrossRef]

39. Stitt, M.; Wilke, I.; Feil, R.; Heldt, H.W. Coarse control of sucrose-phosphate synthase in leaves: Alterations of the kinetic properties in response to the rate of photosynthesis and the accumulation of sucrose. Planta 1988, 174, 217-230. [CrossRef]

40. Signora, L.; Galtier, N.; Skøt, L.; Lucas, H.; Foyer, C.H. Over-expression of sucrose phosphate synthase in Arabidopsis thaliana results in increased foliar sucrose/starch ratios and favours decreased foliar carbohydrate accumulation in plants after prolonged growth with $\mathrm{CO}_{2}$ enrichment. J. Exp. Bot. 1998, 49, 669-680. [CrossRef]

41. Park, J.Y.; Canam, T.; Kang, K.Y.; Ellis, D.D.; Mansfield, S.D. Over-expression of an arabidopsis family A sucrose phosphate synthase (SPS) gene alters plant growth and fibre development. Transgenic Res. 2008, 17, 181-192. [CrossRef]

42. Farcuh, M.; Li, B.; Rivero, R.M.; Shlizerman, L.; Sadka, A.; Blumwald, E. Sugar metabolism reprogramming in a non-climacteric bud mutant of a climacteric plum fruit during development on the tree. J. Exp. Bot. 2017, 68, 5813-5828. [CrossRef]

43. Opassiri, R.; Pomthong, B.; Onkoksoong, T.; Akiyama, T.; Esen, A.; Ketudat Cairns, J.R. Analysis of rice glycosyl hydrolase family 1 and expression of Os4bglu12 $\beta$-glucosidase. BMC Plant Biol. 2006, 6, 33. [CrossRef]

44. Seung, D.; Soyk, S.; Coiro, M.; Maier, B.A.; Eicke, S.; Zeeman, S.C. PROTEIN TARGETING TO STARCH is required for localising GRANULE-BOUND STARCH SYNTHASE to starch granules and for normal amylose synthesis in Arabidopsis. PLoS Biol. 2015, 13, e1002080. [CrossRef]

45. Smith, S.M.; Fulton, D.C.; Chia, T.; Thorneycroft, D.; Chapple, A.; Dunstan, H.; Hylton, C.; Zeeman, S.C.; Smith, A.M. Diurnal changes in the transcriptome encoding enzymes of starch metabolism provide evidence for both transcriptional and posttranscriptional regulation of starch metabolism in Arabidopsis leaves. Plant Physiol. 2004, 136, 2687-2699. [CrossRef]

46. Kajiura, H.; Takata, H.; Akiyama, T.; Kakutani, R.; Furuyashiki, T.; Kojima, I.; Harui, T.; Kuriki, T. In vitro synthesis of glycogen: The structure, properties, and physiological function of enzymatically-synthesized glycogen. Biologia 2011, 66, 387-394. [CrossRef]

47. Li, W.; Li, C.; Gu, Z.; Qiu, Y.; Cheng, L.; Hong, Y.; Li, Z. Retrogradation behavior of corn starch treated with 1,4- $\alpha$-glucan branching enzyme. Food Chem. 2016, 203, 308-313. [CrossRef]

48. Chen, M.H.; Huang, L.F.; Li, H.M.; Chen, Y.R.; Yu, S.M. Signal peptide-dependent targeting of a rice $\alpha$-amylase and cargo proteins to plastids and extracellular compartments of plant cells. Plant Physiol. 2004, 135, 1367-1377. [CrossRef] 
49. Chary, S.N.; Hicks, G.R.; Choi, Y.G.; Carter, D.; Raikhel, N.V. Trehalose-6-phosphate synthase/phosphatase regulates cell shape and plant architecture in Arabidopsis. Plant Physiol. 2008, 146, 97-107. [CrossRef]

50. Mo, Z.; Feng, G.; Su, W.; Liu, Z.; Peng, F. Transcriptomic analysis provides insights into grafting union development in pecan (Carya illinoinensis). Genes 2018, 9, 71. [CrossRef]

51. Osuna, D.; Usadel, B.; Morcuende, R.; Gibon, Y.; Blasing, O.E.; Hohne, M.; Gunter, M.; Kamlage, B.; Trethewey, R.; Scheible, W.R.; et al. Temporal responses of transcripts, enzyme activities and metabolites after adding sucrose to carbon-deprived Arabidopsis seedlings. Plant J. 2007, 49, 463-491. [CrossRef]

52. Paul, M.; Pellny, T.; Goddijn, O. Enhancing photosynthesis with sugar signals. Trends Plant Sci. 2001, 6, 197-200. [CrossRef]

53. Dai, N.; Petreikov, M.; Portnoy, V.; Katzir, N.; Pharr, D.M.; Schaffer, A.A. Cloning and expression analysis of a UDPgalactose/glucose pyrophosphorylase from melon fruit provides evidence for the major metabolic pathway of galactose metabolism in raffinose oligosaccharide metabolizing plants. Plant Physiol. 2006, 142, 294-304. [CrossRef]

54. Nishizawa, A.; Yabuta, Y.; Shigeoka, S. Galactinol and raffinose constitute a novel function to protect plants from oxidative damage. Plant Physiol. 2008, 147, 1251-1263. [CrossRef]

55. Puranik, S.; Sahu, P.P.; Srivastava, P.S.; Prasad, M. NAC proteins: Regulation and role in stress tolerance. Trends Plant Sci. 2012, 17, 369-381. [CrossRef]

56. Liu, X.; Wang, T.; Bartholomew, E.; Black, K.; Dong, M.; Zhang, Y.; Yang, S.; Cai, Y.; Xue, S.; Weng, Y.; et al. Comprehensive analysis of NAC transcription factors and their expression during fruit spine development in cucumber (Cucumis sativus L.). Hortic. Res. 2018, 5, 31. [CrossRef]

57. Jia, D.; Jiang, Z.; Fu, H.; Chen, L.; Liao, G.; He, Y.; Huang, C.; Xu, X. Genome-wide identification and comprehensive analysis of NAC family genes involved in fruit development in kiwifruit (Actinidia). BMC Plant Biol. 2021, 21, 44. [CrossRef] 